\title{
On the gradient set of Lipschitz maps
}

\section{Journal Article}

\section{Author(s):}

Kirchheim, Bernd; Szèkelyhidi, Làszló

Publication date:

2008

Permanent link:

https://doi.org/10.3929/ethz-b-000046131

\section{Rights / license:}

In Copyright - Non-Commercial Use Permitted

\section{Originally published in:}

Journal für die reine und angewandte Mathematik 625, https://doi.org/10.1515/CRELLE.2008.095 


\title{
On the gradient set of Lipschitz maps
}

\author{
By Bernd Kirchheim at Oxford and László Székelyhidi Jr. at Zürich
}

\begin{abstract}
We prove that the essential range of the gradient of planar Lipschitz maps has a connected rank-one convex hull. As a corollary, in combination with the results in [7] we obtain a complete characterization of incompatible sets of gradients for planar maps in terms of rank-one convexity.
\end{abstract}

\section{Introduction}

This paper is concerned with the range of gradients of Lipschitz maps. Let $\Omega \subset \mathbb{R}^{n}$ be a bounded open and connected set, and let $u: \Omega \subset \mathbb{R}^{n} \rightarrow \mathbb{R}^{m}$ be a Lipschitz map. We denote by $[D u]$ the essential range of the gradient of $u$, i.e. the smallest closed subset of $\mathbb{R}^{m \times n}$ such that $D u(x) \in[D u]$ for almost every $x \in \Omega$. Our aim is to find geometric restrictions on, or characterizations of the essential range of gradients of Lipschitz maps.

This issue plays a central role in the study of material microstructure [2], [3], [5], [9], and is linked to the question of existence and regularity of solutions to partial differential inclusions of the type

$$
D u(x) \in K \quad \text { a.e. } x \in \Omega,
$$

where $K \subset \mathbb{R}^{m \times n}$ is a prescribed (compact) set of matrices.

The following construction is well known: let $A, B \in \mathbb{R}^{m \times n}$ be two matrices such that $\operatorname{rank}(A-B)=1$, so that $A-B=a \otimes v$ for some vectors $a \in \mathbb{R}^{m}$ and $v \in \mathbb{R}^{n}$. For any Lipschitz "profile" $h: \mathbb{R} \rightarrow \mathbb{R}$ with $h^{\prime}(t) \in\{0,1\}$ a.e., the map

$$
u(x)=B x+a h(x \cdot v)
$$

is a Lipschitz map whose gradient takes the values $A$ or $B$ almost everywhere. This type of example is called a simple laminate, and whenever two matrices $A, B$ satisfy $\operatorname{rank}(A-B)=1$, one speaks of a rank-one connection (or, more classically, $A$ and $B$ are said to satisfy the Hadamard jump condition). On the other hand, it is also well known that if $A, B \in \mathbb{R}^{m \times n}$ with $\operatorname{rank}(A-B)>1$, then the only Lipschitz maps with gradient $D u(x) \in\{A, B\}$ a.e. are affine maps. Moreover, in [2] J. M. Ball and R. D. James established the much stronger statement that whenever $\left\{u_{j}\right\}$ is a sequence of maps bounded 
in $W^{1,1}$ such that $\operatorname{dist}\left(D u_{j},\{A, B\}\right) \rightarrow 0$ in $L^{1}$ strongly, then-up to a subsequence$D u_{j} \rightarrow A$ or $D u_{j} \rightarrow B$ strongly in $L^{1}$.

A general question, that has received considerable attention recently, is to understand to what extent the above construction is universal. In other words to understand to what extent the presence of rank-one connections is necessary in the essential range of gradients of Lipschitz maps. To put this question into proper perspective, we need to recall the example given by the first author together with D. Preiss of a Lipschitz map $u: \Omega \subset \mathbb{R}^{2} \rightarrow \mathbb{R}^{2}$, where $[D u]$ consists of 5 matrices, none of which are rank-one connected to each other $([12]$, Chapter 4, see also [11] for similar examples). This example shows that it may happen that the set $[D u]$ itself contains no rank-one connections. On the other hand, the construction of the mapping itself relies very much on the presence of rank-one segments in the sense that it proceeds via a (Baire category) variant of an iteration scheme known as convex integration (see [13] for a survey of the theory). In technical terms one key ingredient for this construction to work is that the rank-one convex hull $[D u]^{\text {rc }}$ is a connected set, which contains many rank-one segments in the sense that for any matrix $A \in[D u]^{\mathrm{rc}} \backslash[D u]$ there exists a rank-one segment through $A$ contained in $[D u]^{\text {rc }}$. In other words, although the iterative process of convex integration can eliminate rank-one connections in the essential range $[D u]$ of the limit, the "trail" it leaves behind is a large rank-one convex hull.

Our main result shows that for planar maps this is in some sense optimal:

Theorem 1. Let $\Omega \subset \mathbb{R}^{2}$ be a bounded open and connected set, and $u: \Omega \rightarrow \mathbb{R}^{2} a$ Lipschitz map. Then the rank-one convex hull $[\mathrm{Du}]^{\mathrm{rc}}$ of the essential range of the gradient is connected.

It is important to note that connectedness itself does not imply that $[D u]^{\text {rc }}$ contains rank-one segments. The standard example is simply a planar conformal map. However, in some sense this is the only example. Indeed, if $[D u]^{\text {rc }}$ is connected and contains no rank-one connections, then in fact the differential inclusion

$$
D u(x) \in[D u]^{\mathrm{rc}} \quad \text { for a.e. } x \in \Omega
$$

can be viewed as a (possibly degenerate) elliptic system (see [23], [25]). In particular, we have the following statement:

Corollary 1. If the essential range of the gradient of a Lipschitz map $u: \Omega \subset \mathbb{R}^{2} \rightarrow \mathbb{R}^{2}$ contains an isolated matrix $A \in[D u]$, then there exists another matrix $B \in[D u]^{\mathrm{rc}} \backslash\{A\}$ such that $\operatorname{rank}(A-B)=1$.

We emphasize that Theorem 1 and Corollary 1 are very specific for planar mappings, and the analogue statements are false in higher dimensions in general (see for example [9]).

Our Theorem 1 has interesting implications concerning the study of incompatible sets of gradients. In combination with the results in [6] and [7] we obtain the following theorem.

Theorem 2. Let $K_{1}, K_{2} \subset \mathbb{R}^{2 \times 2}$ be disjoint compact sets which are rank-one incompatible in the sense that

$$
K_{1}^{\mathrm{rc}} \cap K_{2}^{\mathrm{rc}}=\emptyset \quad \text { and } \quad K_{1}^{\mathrm{rc}} \cup K_{2}^{\mathrm{rc}}=\left(K_{1} \cup K_{2}\right)^{\mathrm{rc}} .
$$


Then for any bounded open and connected set $\Omega \subset \mathbb{R}^{2}$ with Lipschitz boundary, and any $p \in[1, \infty)$ there exists a constant $C=C(p, \Omega)$ such that

$$
\min \left\{\int_{\Omega} \operatorname{dist}^{p}\left(D u, K_{1}\right), \int_{\Omega} \operatorname{dist}^{p}\left(D u, K_{2}\right)\right\} \leqq C \int_{\Omega} \operatorname{dist}^{p}\left(D u, K_{1} \cup K_{2}\right)
$$

for all $u \in W^{1, p}\left(\Omega, \mathbb{R}^{2}\right)$.

\section{Outline of the proofs and some preliminaries}

In the proof of Theorem 1 we follow the approach of [7], which is based on the geometric characterization of incompatibility for laminates via a separating curve, introduced by the second author in [24]. We recall from [24] that a continuous, closed curve $\Gamma: \mathscr{S}^{1} \rightarrow \mathbb{R}^{2 \times 2}$ without self-intersetions is said to be a separating curve for a compact set $K \subset \mathbb{R}^{2 \times 2}$ if

$$
K \subset U_{\Gamma}:=\left\{A \in \mathbb{R}^{2 \times 2}: \operatorname{det}(A-\Gamma(t))>0 \text { for all } t \in \mathscr{S}^{1}\right\}
$$

and $K$ is contained in more than one connected component of $U_{\Gamma}$ (the definition implicitly assumes that $U_{\Gamma}$ consists of more than one connected component). In [24], Theorem 4, it is proved that if $K$ contains no rank-one connections and no $T_{4}$ configurations, then such a separating curve exists (upto a change of sign). In turn, the arguments in [24] are used to show in [7], Section 4 , that if $K^{\text {rc }}$ is disconnected, then - upto a change of sign $-K$ admits such a separating curve. A further argument can then be used to refine the choice of curve, so as to obtain an elliptic separating curve. That is, such that for some $\mathscr{K} \geqq 1$

$$
\begin{gathered}
\|\Gamma(t)-\Gamma(s)\|^{2} \leqq \mathscr{K} \operatorname{det}(\Gamma(t)-\Gamma(s)) \text { for all } t, s \in \mathscr{S}^{1}, \\
K \subset \mathscr{E}_{\Gamma}:=\left\{A \in \mathbb{R}^{2 \times 2}:\|A-\Gamma(t)\|^{2}<\mathscr{K} \operatorname{det}(A-\Gamma(t)) \text { for all } t \in \mathscr{S}^{1}\right\},
\end{gathered}
$$

and $K$ is contained in more than one connected component of $\mathscr{E} \Gamma$. In particular one obtains the following

Theorem 3 ([7], Theorem 5). Suppose $K \subset \mathbb{R}^{2 \times 2}$ is a compact set such that $K^{\mathrm{rc}}$ is not connected. Then, possibly after changing sign, there exists an elliptic separating curve for $K$.

Concerning the geometry of $\mathscr{E}_{\Gamma}$ we recall also (cf. [7], Lemma 2) that in fact condition (1) implies that $\mathscr{E}_{\Gamma}$ has precisely two connected components, that are characterized by their projections onto rank-one planes. More precisely, given a unit vector $e \in \mathbb{R}^{2}$, the curve $\Gamma(\cdot) e \subset \mathbb{R}^{2}$ is a Jordan curve, so that $\mathbb{R}^{2} \backslash \Gamma(\cdot) e$ consists of precisely two connected components $\omega^{0}, \omega^{1}$, and we have

Lemma 1 ([7], Lemma 2).

$$
\mathscr{E}_{\Gamma}=\mathscr{E}_{\Gamma}^{0} \cup \mathscr{E}_{\Gamma}^{1}
$$

where

$$
\mathscr{E}_{\Gamma}^{v}=\left\{A \in \mathscr{E}_{\Gamma}: A e \in \omega^{v}\right\} \quad \text { for } v=0,1
$$


In the current paper we take these as our starting point. Thus, if $[D u]^{\text {rc }}$ is disconnected, we apply Theorem 3 with $K=[D u]$ to find the existence of an elliptic separating curve $\Gamma: \mathscr{S}^{1} \rightarrow \mathbb{R}^{2 \times 2}$. As in [7] we interpret the inclusion $[D u] \subset \mathscr{E}_{\Gamma}$ as saying that the maps

$$
u^{t}(x)=u(x)-\Gamma(t) x
$$

are $\mathscr{K}$-quasiregular. However, in our case we have no control over the boundary values of $u^{t}$, hence we cannot conclude that these maps are homeomorphisms. In general they may have branch points. Our strategy is to prove that the set of branch points is in fact independent of $t \in \mathscr{S}^{1}$, and therefore cannot disconnect $\Omega$. In this way we will be able to conclude the incompatibility just as in [7], Theorem 4.

Our paper is organized as follows. In Section 3 we prove a general result about stability of the branch set of quasiregular mappings in $\mathbb{R}^{n}$, and show in Proposition 2 how it can be used to prove separation results for gradients of Lipschitz maps in $\mathbb{R}^{n}$. Then in Section 4 we utilize the stability result together with the existence of a separating curve in case $[D u]^{\text {rc }}$ is disconnected to prove Theorem 1 and Corollary 1.

Finally, in Section 5 we discuss the implications of Theorem 1 to the study of incompatible sets of gradients and in particular the proof of Theorem 2. As the explanation of these implications requires introducing the language of gradient Young measures which does not otherwise play a central role in our paper, we defer the statements and proofs until that section.

\section{Stability of the branch set}

In the following, we will call a connected open subset $\Omega \subset \mathbb{R}^{n}$ a domain. Given a domain $\Omega \subset \mathbb{R}^{n}$ and an open and discrete mapping $u: \Omega \rightarrow \mathbb{R}^{n}$, we shall write $\mu(y, u, G)$ for the local degree of the mapping at $y \in \mathbb{R}^{n}$ with respect to $G$ (provided $y \notin u(\partial G)$ ), $N(y, u, G)=\operatorname{card} u^{-1}(y) \cap G, N(u, G)=\sup _{y} N(y, u, G)$ and $\mathrm{i}(x, u)$ for the local index of $u$ at $x \in \Omega$. We recall that a domain $D \subset \Omega$ is called a normal domain for the mapping $u$ if $u(\partial D)=\partial u(D)$ (note that $\partial u(D) \subset u(\partial D)$ follows automatically from openness of the map). A normal neighbourhood $D$ of $x \in \Omega$ is a normal domain such that $D \cap u^{-1}(u(x))=\{x\}$.

A map $u: \Omega \rightarrow \mathbb{R}^{n}$ is said to be quasiregular, if for some constant $\mathscr{K} \geqq 1$

$$
\|D u(x)\|^{n} \leqq \mathscr{K} \operatorname{det} D u(x) \quad \text { for a.e. } x \in \Omega
$$

where $\|D u(x)\|$ denotes the operator norm of the matrix $D u(x)$. It is well known since the pioneering work of Y. G. Reshetnyak that non-constant quasiregular mappings are open and discrete. The branch set $B(u)$ is defined as the set of points $x \in \Omega$ where $u$ is not locally homeomorphic, that is,

$$
B(u):=\{x \in \Omega: \mathrm{i}(x, u)>1\} .
$$


In particular for quasiregular maps $B(u)$ is a closed set of topological dimension $(n-2)$ and Lebesgue measure zero [20]. For the basic theory of quasiregular mappings, and their topological properties, we refer the reader to [21].

Proposition 1. Let $u: \Omega \rightarrow \mathbb{R}^{n}$ be a $\mathscr{K}$-quasiregular mapping such that $\|D u(x)\| \geqq \varepsilon$ for a.e. $x \in \Omega$, let $G \subset \Omega$ be a subdomain with $\bar{G} \subset \Omega$, and assume that $M:=N(u, G)<\infty$.

Then there exists a constant $\delta=\delta(\varepsilon, \mathscr{K}, M, n)>0$ and for each $x_{0} \in G$ a radius $r\left(x_{0}\right)>0$ so that for any Lipschitz mapping $\phi: \Omega \rightarrow \mathbb{R}^{n}$ with $\|D \phi\|_{\infty}<\delta$,

$$
\min _{\left|x-x_{0}\right|=r}\left|u^{t}(x)-u^{t}\left(x_{0}\right)\right| \geqq \delta r \quad \text { for all } r<r\left(x_{0}\right), t \in[0,1],
$$

where $u^{t}=u+t \phi$. In particular

$$
\mathrm{i}\left(x_{0}, u\right)=\mathrm{i}\left(x_{0}, u+\phi\right) \quad \text { for all } x_{0} \in G
$$

and $B(u) \cap G=B(u+\phi) \cap G$.

Proof. From [19] for every $x_{0} \in G$ there exists a radius $r\left(x_{0}\right)>0$ so that $B_{r\left(x_{0}\right)}\left(x_{0}\right) \subset \Omega$, and for $r<r\left(x_{0}\right)$

$$
\max _{\left|x-x_{0}\right|=r}\left|u(x)-u\left(x_{0}\right)\right| \leqq L \min _{\left|x-x_{0}\right|=r}\left|u(x)-u\left(x_{0}\right)\right|,
$$

where $L=L(\mathscr{K}, M, n)$. Moreover,

$$
\int_{B_{r}\left(x_{0}\right)} \operatorname{det} D u(x) d x=\int_{\mathbb{R}^{n}} N\left(y, u, B_{r}\left(x_{0}\right)\right) d y \leqq M\left|u\left(B_{r}\left(x_{0}\right)\right)\right|,
$$

and on the other hand $|\operatorname{det} D u(x)| \geqq \mathscr{K}^{-1} \varepsilon^{n}$. Hence

$$
\frac{1}{M \mathscr{K}} \varepsilon^{n} \leqq \frac{\left|u\left(B_{r}\left(x_{0}\right)\right)\right|}{\left|B_{r}\left(x_{0}\right)\right|} .
$$

In particular we deduce that for all $r<r\left(x_{0}\right)$

$$
\frac{1}{L(M \mathscr{K})^{1 / n}} \varepsilon r \leqq \min _{\left|x-x_{0}\right|=r}\left|u(x)-u\left(x_{0}\right)\right|
$$

For $t \in[0,1]$ define $u^{t}=u+t \phi$. Then

$$
u^{t}(x)-u^{t}\left(x_{0}\right)=u(x)-u\left(x_{0}\right)+t\left(\phi(x)-\phi\left(x_{0}\right)\right)
$$

so that

$$
\min _{\left|x-x_{0}\right|=r}\left|u^{t}(x)-u^{t}\left(x_{0}\right)\right| \geqq\left(\frac{1}{L(M \mathscr{K})^{1 / n}} \varepsilon-t \delta\right) r .
$$

Choosing $\delta=\frac{1}{2} \frac{1}{L(M \mathscr{K})^{1 / n}} \varepsilon$ we find

$$
\min _{\left|x-x_{0}\right|=r}\left|u^{t}(x)-u^{t}\left(x_{0}\right)\right| \geqq \delta r \quad \text { for all } r<r\left(x_{0}\right), t \in[0,1],
$$


and in particular $u^{t}(x) \neq u^{t}\left(x_{0}\right)$ for all $x \in \partial B_{r}\left(x_{0}\right)$. Hence

$$
\mu\left(u^{0}\left(x_{0}\right), u^{0}, B_{r}\left(x_{0}\right)\right)=\mu\left(u^{1}\left(x_{0}\right), u^{1}, B_{r}\left(x_{0}\right)\right) \text { for all } 0<r<r\left(x_{0}\right) \text {. }
$$

For $x_{0} \in \Omega$ the local topological index of the mapping $u$ at $x_{0}$ is defined to be

$$
\mathrm{i}\left(x_{0}, u\right)=\mu\left(u\left(x_{0}\right), u, B_{r}\left(x_{0}\right)\right),
$$

where $r>0$ is chosen sufficiently small so that $\overline{B_{r}\left(x_{0}\right)} \cap u^{-1}\left\{u\left(x_{0}\right)\right\}=\left\{x_{0}\right\}$. Therefore (4) implies that

$$
\mathrm{i}\left(x_{0}, u\right)=\mathrm{i}\left(x_{0}, u+\phi\right) \text {. }
$$

Since the branch set is defined as $B(u)=\{x \in \Omega: \mathrm{i}(x, u)>1\}$, we deduce that $B(u) \cap G=B(u+\phi) \cap G$. Q.E.D.

Proposition 2. Let $\Gamma \subset \mathbb{R}^{n \times n}$ be a compact set of $n \times n$ matrices and $\Omega \subset \mathbb{R}^{n} a$ domain. Let $u \in W^{1, n}\left(\Omega, \mathbb{R}^{n}\right)$, and suppose that there exists $\mathscr{K} \geqq 1$ and $\varepsilon>0$ such that for all $A \in \Gamma$

$$
\varepsilon \leqq\|D u(x)-A\|^{n} \leqq \mathscr{K} \operatorname{det}(D u(x)-A) \text { a.e. } x \in \Omega
$$

Then there exists an open and connected subset $\Omega_{0} \subset \Omega$ with $\left|\Omega \backslash \Omega_{0}\right|=0$ such that for all $x_{0} \in \Omega_{0}$ there exists a radius $\tilde{r}\left(x_{0}\right)>0$ such that

$$
u(x)-u(y) \neq A(x-y) \quad \text { for all } x, y \in B_{\tilde{r}\left(x_{0}\right)}\left(x_{0}\right) \text { and all } A \in \Gamma \text {. }
$$

Proof. For simplicity of notation let us treat $\Gamma \subset \mathbb{R}^{n \times n}$ as the image of a continuous map $\Gamma: \mathscr{S} \rightarrow \mathbb{R}^{n \times n}$, where $\mathscr{S}$ is a compact metric space which we think of as andex set. Consider for any $t \in \mathscr{S}$ the mapping

$$
u^{t}(x):=u(x)-\Gamma(t) x .
$$

By assumption $u^{t} \in W^{1, n}\left(\Omega, \mathbb{R}^{n}\right)$ and

$$
\varepsilon \leqq\left\|D u^{t}(x)\right\|^{n} \leqq \mathscr{K} \operatorname{det} D u^{t}(x) \quad \text { a.e. } x \in \Omega
$$

in particular $\left\{u^{t}\right\}_{t \in \mathscr{S}}$ is an equicontinuous family of quasiregular mappings.

Let $G \subset \Omega$ be a subdomain with compact closure and such that $\bar{G} \subset \Omega$ and $|\partial G|=0$. From [18] we know that $N\left(u^{t}, G\right)<\infty$ for each $t \in \mathscr{S}$. We aim to show that in fact $\sup _{t \in \mathscr{S}} N\left(u^{t}, G\right)<\infty$. To this end note that since each $u^{t}$ is a discrete mapping, for each $x \in G$ and each $t \in \mathscr{S}$ there exists $r=r(x, t)>0$ so that

$$
\overline{B_{r}(x)} \cap\left(u^{t}\right)^{-1}\left\{u^{t}(x)\right\}=\{x\} .
$$

More precisely from Proposition 1 we deduce that there exists $r=r(x, t)>0$ and $\delta=\delta(t)>0$ so that

$$
\overline{B_{r}(x)} \cap\left(u^{s}\right)^{-1}\left\{u^{s}(x)\right\}=\{x\} \quad \text { for all }|s-t|<\delta
$$


and

$$
\operatorname{dist}\left(u^{s}(x), u^{s}\left(\partial B_{r}(x)\right)\right) \geqq \delta r \quad \text { for all }|s-t|<\delta
$$

holds for all $t \in \mathscr{S}$. Hence by compactness of $\mathscr{S}$ there exists $r=r(x)>0$ and $\delta>0$ (now independent of $t$ ) so that

$$
\overline{B_{r}(x)} \cap\left(u^{t}\right)^{-1}\left\{u^{t}(x)\right\}=\{x\} \text { for all } t \in \mathscr{S}
$$

and

$$
\operatorname{dist}\left(u^{t}(x), u^{t}\left(\partial B_{r}(x)\right)\right) \geqq \delta r \quad \text { for all } t \in \mathscr{S} \text {. }
$$

Indeed, the sets $V(t):=\{s \in \mathscr{S}:|t-s|<\delta(t)\}$ form an open cover for $\mathscr{S}$, so it suffices to take a finite subcover $V\left(t_{1}\right), \ldots, V\left(t_{N}\right)$ and then define

$$
r(x)=\min _{i=1, \ldots, N} r\left(x, t_{i}\right) \quad \text { and } \quad \delta=\min _{i=1, \ldots, N} \delta\left(t_{i}\right)
$$

in (5) and (6). Let

$$
s(x, t):=\operatorname{dist}\left(u^{t}(x), u^{t}\left(\partial B_{r}(x)\right)\right),
$$

and let $U(x, t)$ be the connected component of

$$
\left(u^{t}\right)^{-1}\left(B_{s(x, t)}\left(u^{t}(x)\right)\right)
$$

containing $x$. Then $U(x, t) \subset B_{r(x)}(x)$ is a normal neighbourhood of $x$ for the mapping $u^{t}$. Since the family $\left\{u^{t}\right\}$ is equicontinuous, from (6) we deduce that there exists $\tilde{r}=\tilde{r}(x)>0$ so that $B_{\tilde{r}}(x) \subset U(x, t)$. Since $\tilde{r}(x)$ is independent of $t$, there exists a number $J \in \mathbb{N}$ so that for each fixed $t \in \mathscr{S}$ the compact set $\bar{G}$ can be covered by at most $J$ normal neighbourhoods $U\left(x_{1}, t\right), \ldots, U\left(x_{J}, t\right)$. Then

$$
N\left(u^{t}, G\right) \leqq \sum_{j=1}^{J} N\left(u^{t}, U\left(x_{j}, t\right)\right)=\sum_{j=1}^{J} i\left(x_{j}, u^{t}\right)
$$

On the other hand Proposition 1 implies that for each fixed $x_{0} \in G$ the function $t \mapsto i\left(x_{0}, u^{t}\right)$ is continuous, hence bounded on $\mathscr{S}$. Therefore we deduce that $N\left(u^{t}, G\right)$ is bounded independently of $t$.

Proposition 1 now implies that there exists $\delta>0$ (not depending on $t$ ) so that

$$
B\left(u^{t}\right) \cap G=B\left(u^{s}\right) \cap G \text { for all } s, t \in \mathscr{S} \text { with }|s-t|<\delta .
$$

In particular the set

$$
B=\bigcup_{t \in \mathscr{S}} B\left(u^{t}\right) \cap G
$$

is a finite union of closed sets of topological dimension $(n-2)$ and Lebesgue measure zero [20], hence $B$ is a closed set of dimension $(n-2)$ and Lebesgue measure zero. This implies that the set $G_{0}:=G \backslash B$ is open and connected (see [8], Theorem IV.4), and $\left|G \backslash G_{0}\right|=0$. 
In $G_{0}$ each mapping $u^{t}$ is a local homeomorphism. More precisely, let $x_{0} \in G_{0}$. Since $U\left(x_{0}, t\right)$ is a normal neighbourhood of $x_{0}$ for the mapping $u^{t}$, we have

$$
N\left(u^{t}, U\left(x_{0}, t\right)\right)=\mathrm{i}\left(x_{0}, u^{t}\right)=1 \quad \text { for all } t \in \mathscr{S}
$$

Since $B_{\tilde{r}\left(x_{0}\right)}\left(x_{0}\right) \subset U\left(x_{0}, t\right)$ for all $t \in \mathscr{S}$, we deduce that each mapping $u^{t}$ is injective on $B_{\tilde{r}\left(x_{0}\right)}\left(x_{0}\right)$. Therefore

$$
u^{t}(x) \neq u^{t}(y) \quad \text { for all } t \in \mathscr{S}, x, y \in B_{\tilde{r}\left(x_{0}\right)}\left(x_{0}\right)
$$

in other words

$$
u(x)-u(y)-A(x-y) \neq 0 \quad \text { for all } x, y \in B_{\tilde{r}\left(x_{0}\right)}\left(x_{0}\right) \text { and all } A \in \Gamma .
$$

The proposition now follows by exhausting $\Omega$ with a nested sequence of bounded subdomains $G \subset \Omega$ with $|\partial G|=0$ and $\bar{G} \subset \Omega$. Q.E.D.

\section{Proof of the main result}

Proof of Theorem 1. Let $K=[D u]$. Recall, that by definition $[D u]$ is the smallest closed subset of $\mathbb{R}^{2 \times 2}$ such that $D u(x) \in[D u]$ for almost every $x \in \Omega$.

We argue by contradiction, assuming that $K^{\mathrm{rc}}$ is not connected. According to Theorem 3 we may assume that there exists an elliptic separating curve for $K$, i.e. a continuous closed curve $\Gamma: \mathscr{S}^{1} \rightarrow \mathbb{R}^{2 \times 2}$ without self-intersections such that (1) and (2) hold, and $K$ is contained in more than one component of $\mathscr{E}_{\Gamma}$. Since $K$ and $\Gamma$ are compact, there exists $\varepsilon>0$ such that for all $t \in \mathscr{S}^{1}$

$$
\varepsilon \leqq\|D u(x)-\Gamma(t)\|^{2} \leqq \mathscr{K} \operatorname{det}(D u(x)-\Gamma(t)) \quad \text { a.e. } x \in \Omega
$$

But then Proposition 2 implies that there exists a connected and open subset $\Omega_{0} \subset \Omega$ with $\left|\Omega \backslash \Omega_{0}\right|=0$ and for each $x_{0} \in \Omega_{0}$ there exists a radius $\tilde{r}\left(x_{0}\right)>0$ such that

$$
u(x)-u(y) \neq \Gamma(t)(x-y) \quad \text { for all } x, y \in B_{\tilde{r}\left(x_{0}\right)}\left(x_{0}\right) \text { and } t \in \mathscr{S}^{1} .
$$

Setting $y=x+\delta e_{1}$ for $0<\delta<\tilde{r}(x)$ we obtain

$$
\frac{u\left(x+\delta e_{1}\right)-u(x)}{\delta} \neq \Gamma(t) e_{1} \quad \text { for all } t \in \mathscr{S}^{1},(x, \delta) \in \Delta
$$

where $\Delta=\left\{(x, \delta): x \in \Omega_{0}, 0<\delta<\tilde{r}(x)\right\}$. Since $\Gamma$ satisfies (1),

$$
\Gamma(\cdot) e_{1}: \mathscr{S}^{1} \rightarrow \mathbb{R}^{2}
$$

is a continuous imbedding, hence by the Jordan separation theorem the image $\left\{\Gamma(t) e_{1}: t \in \mathscr{S}^{1}\right\}$ separates $\mathbb{R}^{2}$ into two disjoint regions $\omega$ and $\mathbb{R}^{2} \backslash \bar{\omega}$. Since $\Delta$ is a connected 
set, we deduce that

$$
\begin{aligned}
& \frac{u\left(x+\delta e_{1}\right)-u(x)}{\delta} \in \omega \quad \text { for all }(x, \delta) \in \Delta, \\
& \text { or } \\
& \frac{u\left(x+\delta e_{1}\right)-u(x)}{\delta} \in \mathbb{R}^{2} \backslash \bar{\omega} \text { for all }(x, \delta) \in \Delta .
\end{aligned}
$$

Since $u$ is quasiregular, it is differentiable almost everywhere in $\Omega$. Therefore, recalling (7) and that $\left|\Omega \backslash \Omega_{0}\right|=0$, we obtain

$$
\partial_{x_{1}} u(x) \in \bar{\omega} \text { for a.e. } x \in \Omega,
$$

$$
\begin{gathered}
\text { or } \\
\partial_{x_{1}} u(x) \in \mathbb{R}^{2} \backslash \omega \text { for a.e. } x \in \Omega \text {. }
\end{gathered}
$$

In light of Lemma 1 this implies that $K$ has to be contained in a single component of $\mathscr{E} \Gamma$, giving us the required contradiction. Q.E.D.

Proof of Corollary 1. Suppose that $A \in[D u]$ is an isolated point, and assume for a contradiction that for all $B \in[D u]^{\mathrm{rc}} \backslash\{A\}$ we have $\operatorname{rank}(A-B)>1$.

If $\operatorname{det}(A-B)>0$ for all $B \in[D u] \backslash\{A\}$, then - since $A$ is isolated and hence $[D u] \backslash\{A\}$ is compact-there exists a constant $\gamma \geqq 1$ so that

$$
\|D u(x)-A\|^{2} \leqq \gamma \operatorname{det}(D u(x)-A) \quad \text { a.e. } x \in \Omega .
$$

This means that the map $x \mapsto u(x)-A x$ is quasiregular. By the unique continuation property of quasiregular mappings we deduce that $D u(x)=A x$ a.e., a contradiction. Similarly, we obtain the same contradiction if $\operatorname{det}(A-B)<0$ for all $B \in[D u] \backslash\{A\}$ (by just considering a linear change of variables).

Therefore, we may assume that there exists at least two matrices

$$
A_{1}, A_{2} \in[D u] \backslash\{A\}
$$

such that $\operatorname{det}\left(A-A_{1}\right)<0$ and $\operatorname{det}\left(A-A_{2}\right)>0$. If $[D u]^{\text {rc }} \backslash\{A\}$ is connected, we obtain by continuity the existence of $B \in[D u]^{\mathrm{rc}} \backslash\{A\}$ with $\operatorname{det}(A-B)=0$.

Otherwise let $K_{1}, K_{2}$ be disjoint connected components of $[D u]^{\mathrm{rc}} \backslash\{A\}$ containing $A_{1}$ and $A_{2}$, respectively. We claim first of all that

$$
A \in \bar{K}_{1} \cap \bar{K}_{2}
$$

Indeed, assume the contrary, so that, without loss of generality, $A \notin \bar{K}_{1}$. Then there exists $\eta>0$ with

$$
B_{2 \eta}(A) \cap K_{1}=\emptyset .
$$


As $[D u]^{\text {rc }}$ and hence $\tilde{K}:=[D u]^{\mathrm{rc}} \backslash B_{\eta}(A)$ is compact, and since $K_{1}$ is clearly the connected component of $\tilde{K}$ containing $A_{1}$, we see from [14], S44.II.2, that $K_{1}$ is equal to the intersection of the family $\mathscr{F}$ of all open and closed subsets of $\tilde{K}$ which contain $A_{1}$. In particular, since $\mathscr{F}$ is closed under finite intersections, we conclude that there is some $V \in \mathscr{F}$ with

$$
V \subset B_{\eta}\left(K_{1}\right) \text { and } \quad A_{2} \notin V
$$

Here $B_{\eta}\left(K_{1}\right)$ denotes the open $\eta$-neighbourhood of $K_{1}$. But then $V \cap B_{\eta}(A)=\emptyset$ because of (10), and hence $V$ is closed and open in $[D u]^{\text {rc }}$. We conclude that $[D u]^{\text {rc }}$ would be disconnected, in contradiction with Theorem 1. This proves the claim (9).

Now suppose without loss of generality that $\operatorname{det}\left(A_{1}-A_{2}\right)>0$, and consider the function $f(X)=\operatorname{det}\left(X-A_{1}\right)$ restricted to $K_{2}$. Since $\operatorname{det}\left(A-A_{1}\right)<0$ and $A \in \bar{K}_{2}$, there exists $A^{\prime} \in K_{2}$ such that $f\left(A^{\prime}\right)<0$, by continuity. On the other hand $f\left(A_{2}\right)>0$, therefore there exists, again by continuity, $A_{3} \in K_{2}$ with $f\left(A_{3}\right)=0$. In particular $\left[A_{1}, A_{3}\right]$ is a rank-one segment, which therefore is contained in $[D u]^{\mathrm{rc}}$. If $A \notin\left[A_{1}, A_{3}\right]$ then we obtain a contradiction with the assumption that $A_{1} \in K_{1}$ and $A_{3} \in K_{2}$ are contained in different connected components of $[D u]^{\mathrm{rc}} \backslash\{A\}$. On the other hand, if $A \in\left[A_{1}, A_{3}\right]$, then in particular $\operatorname{det}\left(A-A_{1}\right)=0$, contradicting the assumption that $\operatorname{det}\left(A-A_{1}\right)<0$. This finishes the proof. Q.E.D.

\section{Incompatible sets of gradients}

Following [1] two disjoint compact sets of matrices $K_{1}, K_{2} \subset \mathbb{R}^{m \times n}$ are said to be incompatible if whenever $\Omega$ is a bounded open and connected set and $\left\{u_{j}\right\}$ is a sequence of maps bounded in $W^{1,1}(\Omega)$ such that

$$
\operatorname{dist}\left(D u_{j}, K_{1} \cup K_{2}\right) \rightarrow 0 \quad \text { in } L^{1}(\Omega) \text { strongly, }
$$

then-up to a subsequence-

$$
\operatorname{dist}\left(D u_{j}, K_{1}\right) \rightarrow 0 \quad \text { or } \quad \operatorname{dist}\left(D u_{j}, K_{2}\right) \rightarrow 0 \text { strongly in } L^{1}(\Omega)
$$

In the language of Young measures this is equivalent to saying that whenever $\left\{v_{x}\right\}_{x \in \Omega}$ is a gradient Young measure supported in $K_{1} \cup K_{2}$, that is,

$$
\operatorname{supp} v_{x} \subset K_{1} \cup K_{2} \quad \text { a.e. } x \in \Omega,
$$

then

$$
\text { either } \operatorname{supp} v_{x} \subset K_{1} \text { a.e. } \text { or } \operatorname{supp} v_{x} \subset K_{2} \text { a.e. }
$$

In short, the sets $K_{1}$ and $K_{2}$ are incompatible for gradient Young measures. From the point of view of material microstructure it is of interest to be able to characterize such incompatible sets. Indeed, in this situation the inclusion problem $D u(x) \in K_{1} \cup K_{2}$ would correspond to energy-minimizing deformations of an elastic material, and roughly speaking incompatibility prevents large scale oscillations (oscillations between $K_{1}$ and $K_{2}$ ), whilst still allowing for local oscillations within each individual energy-well $K_{1}$ or $K_{2}$. 
Pairs of incompatible sets have several nice features. First of all, if $K_{1}$ and $K_{2}$ are incompatible for gradient Young measures, then sufficiently small $\varepsilon$-neighbourhoods $\left(K_{1}\right)_{\varepsilon}$ and $\left(K_{2}\right)_{\varepsilon}$ are still incompatible. This was established by Ball and James in the early 90s in their study of metastability [1]. Moreover, one gets precise control of the gradient for approximating sequences in the form of a rigidity estimate:

$$
\min \left\{\int_{\Omega} \operatorname{dist}^{p}\left(D u, K_{1}\right), \int_{\Omega} \operatorname{dist}^{p}\left(D u, K_{2}\right)\right\} \leqq C_{p, \Omega} \int_{\Omega} \operatorname{dist}^{p}\left(D u, K_{1} \cup K_{2}\right),
$$

valid for all $u \in W^{1, p}\left(\Omega, \mathbb{R}^{m}\right)$ and all $p \in[1, \infty)$. This was proved in [6] using the method of Ball and James [1].

The simplest example of incompatible sets, as already pointed out in the introduction, is given by the singleton sets $\{A\},\{B\}$ whenever $A, B \in \mathbb{R}^{m \times n}$ with $\operatorname{rank}(A-B)>1$. In [27] $\mathrm{K}$. Zhang showed that in this case there exists $\varepsilon>0$, so that the sets

$$
K_{1}=\left\{X \in \mathbb{R}^{m \times n}:|X-A| \leqq \varepsilon\right\} \quad \text { and } \quad K_{2}=\left\{X \in \mathbb{R}^{m \times n}:|X-B| \leqq \varepsilon\right\}
$$

are still incompatible (in fact Zhang's result applies to the neighbourhood of any finite collection of matrices contained in a subspace without rank-one connections). More precisely, Zhang obtains explicit estimates for $\varepsilon>0$ in terms of Schauder $L^{\infty}-$ BMO estimates (see also [26] for a similar technique applied to incompatible wells in 2D). In contrast, in the aforementioned stability result of Ball and James $\varepsilon>0$ is obtained in a contradiction argument. Other types of explicit examples of incompatible sets were obtained by V. Šverák [22] in connection with the Monge-Ampère equation and by J. P. Matos in [15] concerning the two-well problem in 3D.

Our Theorem 1, combined with results in [7] allows us to completely characterize incompatible sets in $\mathbb{R}^{2 \times 2}$ in terms of the underlying rank-one geometry.

Corollary 2. Two disjoint compact sets $K_{1}, K_{2} \subset \mathbb{R}^{2 \times 2}$ are incompatible for gradient Young measures if and only if $K_{1}^{\mathrm{rc}} \cap K_{2}^{\mathrm{rc}}=\emptyset$ and $K_{1}^{\mathrm{rc}} \cup K_{2}^{\mathrm{rc}}=\left(K_{1} \cup K_{2}\right)^{\mathrm{rc}}$.

In order to explain the meaning of this result, we briefly recall a few more notions from the nonconvex calculus of variations. First of all, a gradient Young measure $\left\{v_{x}\right\}_{x \in \Omega}$ is said to be homogeneous if $v_{x}$ is independent of $x \in \Omega$. Homogeneous gradient Young measures appear in the study of compactness of sequences of gradients $\left\{D u_{j}\right\}$. A further subclass of homogeneous gradient Young measures is formed by laminates. Roughly speaking laminates are probability measures that can be characterized by rankone connections. More precisely, laminates are the smallest class of probability measures on the space of matrices that are

(i) closed under splitting,

(ii) closed under weak* convergence,

(iii) and contain all measures of the form $\lambda \delta_{A}+(1-\lambda) \delta_{B}$ whenever $\operatorname{rank}(A-B) \leqq 1$ and $\lambda \in[0,1]$. 
Being closed under splitting means that if $v$ is a laminate of the form

$$
v=\lambda \delta_{A}+(1-\lambda) \tilde{v}
$$

for some probability measure $\tilde{v}$, and $\mu$ is a laminate with barycenter $\bar{\mu}=A$, then the measure

$$
\lambda \mu+(1-\lambda) \tilde{v}
$$

is also a laminate. For basic properties of these classes of measures we refer the reader to [16], [17].

We recall in particular that the rank-one convex hull $K^{\mathrm{rc}}$ of a compact set of matrices can be defined as the set of barycenters of laminates supported in $K$ :

$$
K^{\mathrm{rc}}=\{\bar{\mu}: \mu \text { is a laminate with } \operatorname{supp} \mu \subset K\} .
$$

To each class of measures one can associate a notion of incompatibility for pairs of compact sets. Thus for example $K_{1}, K_{2}$ are said to be incompatible for laminates if whenever $\mu$ is a laminate with support

$$
\operatorname{supp} \mu \subset K_{1} \cup K_{2}
$$

then

$$
\operatorname{supp} \mu \subset K_{1} \quad \text { or } \quad \operatorname{supp} \mu \subset K_{2} \text {. }
$$

Similarly, $K_{1}, K_{2}$ are said to be homogeneously incompatible if they are incompatible for homogeneous gradient Young measures. Equivalently, $K_{1}, K_{2}$ are homogeneously incompatible if whenever $\left\{u_{j}\right\}$ is a sequence of maps bounded in $W_{0}^{1,1}(\Omega)$ such that

$$
\operatorname{dist}\left(A+D u_{j}, K_{1} \cup K_{2}\right) \rightarrow 0 \quad \text { in } L^{1}(\Omega) \text { strongly }
$$

for some matrix $A$, then-up to a subsequence-

$$
\operatorname{dist}\left(A+D u_{j}, K_{1}\right) \rightarrow 0 \quad \text { or } \quad \operatorname{dist}\left(A+D u_{j}, K_{2}\right) \rightarrow 0 \quad \text { strongly in } L^{1}(\Omega)
$$

The meaning of Corollary 2 is that in the space of $2 \times 2$ matrices the three notions of incompatibility are equivalent: equivalent:

Corollary 3. Let $K_{1}, K_{2} \subset \mathbb{R}^{2 \times 2}$ be disjoint compact sets. The following are

(i) $K_{1}, K_{2}$ are incompatible for gradient Young measures.

(ii) $K_{1}, K_{2}$ are incompatible for homogeneous gradient Young measures.

(iii) $K_{1}, K_{2}$ are incompatible for laminates. 
The equivalence between (ii) and (iii) was already proved in [7], Corollary 1. Here we establish the equivalence of (i) and (ii), assuming that (ii) and (iii) are equivalent. Proving this equivalence amounts to a passage from approximating sequences of the form $\left\{A+D u_{j}\right\}$ with $D u_{j} \in W_{0}^{1,1}(\Omega)$ to general sequences $\left\{D u_{j}\right\} \subset W^{1,1}(\Omega)$. Indeed, a crucial aspect of Theorem 1 is that there is no assumption made on the boundary values of the map $u: \Omega \rightarrow \mathbb{R}^{2}$, and this is the main new aspect of our paper.

Proof of Corollary 2. One direction is easy: if $K_{1}, K_{2}$ are incompatible for gradient Young measures, then in particular they are incompatible for laminates. Thus any laminate $\mu$ with support supp $\mu \subset K_{1} \cup K_{2}$ has to be supported in $K_{1}$ or $K_{2}$. Therefore the definition of rank-one convex hull implies that $\left(K_{1} \cup K_{2}\right)^{\mathrm{rc}}=K_{1}^{\mathrm{rc}} \cup K_{2}^{\mathrm{rc}}$. It remains to show that $K_{1}^{\mathrm{rc}} \cap K_{2}^{\mathrm{rc}}=\emptyset$. Assume for a contradiction that $K_{1}^{\mathrm{rc}} \cap K_{2}^{\mathrm{rc}} \neq \emptyset$, so that there exist laminates $\mu_{1}, \mu_{2}$ with support supp $\mu_{i} \subset K_{i}$ with common barycenter $\overline{\mu_{1}}=\overline{\mu_{2}} \in K_{1}^{\mathrm{rc}} \cap K_{2}^{\mathrm{rc}}$. But then the laminate defined as $\mu:=\frac{1}{2} \mu_{1}+\frac{1}{2} \mu_{2}$ has support $\operatorname{supp} \mu \subset K_{1} \cup K_{2}$, but doesn't satisfy $\operatorname{supp} \mu \subset K_{1}$ or supp $\mu \subset K_{2}$. This gives a contradiction, and therefore necessarily $K_{1}^{\mathrm{rc}} \cap K_{2}^{\mathrm{rc}}=\emptyset$.

For the other direction suppose now that $K_{1}^{\mathrm{rc}} \cap K_{2}^{\mathrm{rc}}=\emptyset$ and $\left(K_{1} \cup K_{2}\right)^{\mathrm{rc}}=K_{1}^{\mathrm{rc}} \cup K_{2}^{\mathrm{rc}}$. We claim that in this case $K_{1}$ and $K_{2}$ are incompatible for laminates. Indeed, suppose $\mu$ is a laminate with support supp $\mu \subset K_{1} \cup K_{2}$. Then

$$
\operatorname{supp} \mu \subset\left(K_{1} \cup K_{2}\right)^{\mathrm{rc}}=K_{1}^{\mathrm{rc}} \cup K_{2}^{\mathrm{rc}},
$$

and on the other hand it is well known that $(\operatorname{supp} \mu)^{\mathrm{rc}}$ is a connected set (see [12], Theorem 4.9). Therefore necessarily

$$
(\operatorname{supp} \mu)^{\mathrm{rc}} \subset K_{1}^{\mathrm{rc}} \quad \text { or } \quad(\operatorname{supp} \mu)^{\mathrm{rc}} \subset K_{2}^{\mathrm{rc}}
$$

To conclude that $\operatorname{supp} \mu \subset K_{1}$ or $\operatorname{supp} \mu \subset K_{2}$ just note that $\operatorname{supp} \mu \subset(\operatorname{supp} \mu)^{\mathrm{rc}}$ and that $K_{i} \cap K_{j}^{\text {rc }}=\emptyset$ for $i \neq j$.

Having just shown that $K_{1}$ and $K_{2}$ are incompatible for laminates, we can now invoke [7], Corollary 1, which implies that $K_{1}$ and $K_{2}$ are incompatible for homogeneous gradient Young measures. Using standard machinery on homogeneous gradient Young measures [10], [16], [17], it follows that $K_{1}^{\mathrm{qc}} \cap K_{2}^{\mathrm{qc}}=\emptyset$ and $\left(K_{1} \cup K_{2}\right)^{\mathrm{qc}}=K_{1}^{\mathrm{qc}} \cup K_{2}^{\mathrm{qc}}$, just as above for the rank-one convex hull (see also [7], Corollary 3 ).

Now suppose that $\left\{v_{x}\right\}_{x \in \Omega}$ is a gradient Young measure such that

$$
\operatorname{supp} v_{x} \subset K_{1} \cup K_{2} \quad \text { for a.e. } x \in \Omega \text {. }
$$

Since $v_{x}$ coincides with a homogeneous gradient Young measure for a.e. $x$ and $K_{1}, K_{2}$ are incompatible for homogeneous gradient Young measures, we deduce that for almost every $x \in \Omega$ there exists $i=i_{x} \in\{1,2\}$ such that

$$
\operatorname{supp} v_{x} \subset K_{i_{x}} \text {. }
$$


It remains to show that $i_{x}=1$ a.e. or $i_{x}=2$ a.e. To this end recall (see [10]) that because $\left\{v_{x}\right\}_{x \in \Omega}$ is a gradient Young measure, there exists a Lipschitz mapping $u: \Omega \rightarrow \mathbb{R}^{2}$ such that $D u(x)=\bar{v}_{x}$ a.e. $x \in \Omega$. In particular

$$
[D u] \subset\left(K_{1} \cup K_{2}\right)^{\mathrm{qc}} .
$$

By Theorem 1 we know that $[D u]^{\text {rc }}$ is connected, and on the other hand

$$
[D u]^{\mathrm{rc}} \subset[D u]^{\mathrm{qc}} \subset\left(K_{1} \cup K_{2}\right)^{\mathrm{qc}}=K_{1}^{\mathrm{qc}} \cup K_{2}^{\mathrm{qc}} .
$$

Since $K_{1}^{\mathrm{qc}} \cap K_{2}^{\mathrm{qc}}=\emptyset$, we deduce that

$$
[D u] \subset K_{1}^{\mathrm{qc}} \quad \text { or } \quad[D u] \subset K_{2}^{\mathrm{qc}} .
$$

Finally, note that $\bar{v}_{x} \in K_{i}^{\mathrm{qc}}$ if and only if $\operatorname{supp} v_{x} \subset K_{i}$ (for $\left.i=1,2\right)$ since $K_{1}^{\mathrm{qc}} \cap K_{2}^{\mathrm{qc}}=\emptyset$. Hence we conclude that $\operatorname{supp} v_{x} \subset K_{1}$ a.e. $x \in \Omega$ or supp $v_{x} \subset K_{2}$ a.e. $x \in \Omega$. Q.E.D.

Proof of Corollary 3. Since the implications (i) $\Rightarrow$ (ii) $\Rightarrow$ (iii) follow from the definitions, it suffices to prove that (iii) $\Rightarrow$ (i). Suppose that $K_{1}, K_{2}$ are incompatible for laminates. Then, precisely as in the proof of Corollary 2 above, we have that $\left(K_{1} \cup K_{2}\right)^{\mathrm{rc}}=K_{1}^{\mathrm{rc}} \cup K_{2}^{\mathrm{rc}}$ and $K_{1}^{\mathrm{rc}} \cap K_{2}^{\mathrm{rc}}=\emptyset$. But then Corollary 2 implies that $K_{1}, K_{2}$ are incompatible for gradient Young measures. Q.E.D.

Proof of Theorem 2. The statement of the theorem is a direct consequence of Corollary 2 together with [6], Theorem 1.2. Q.E.D.

\section{References}

[1] Ball, J. M., and James, R. D., Incompatible sets of gradients and metastability, in preparation.

[2] Ball, J. M., and James, R. D., Fine phase mixtures as minimizers of energy, Arch. Rat. Mech. Anal. 100 (1987), 13-52.

[3] Bhattacharya, K., Firoozye, N. B., James, R. D., and Kohn, R. V., Restrictions on microstructure, Proc. Roy. Soc. Edinburgh Sect. A 124, 5 (1994), 843-878.

[4] Bourbaki, N., General topology, Chapters 1-4, Elements of Mathematics, Springer-Verlag, Berlin 1998. Translated from the French, Reprint of the 1989 English translation.

[5] Chipot, M., and Kinderlehrer, D., Equilibrium configurations of crystals, Arch. Rat. Mech. Anal. 103 (1988), 237-277.

[6] De Lellis, C., and Székelyhidi, Jr., L., Simple proof of two-well rigidity, C. R. Math. Acad. Sci. Paris 343, 5 (2006), 367-370.

[7] Faraco, D., and Székelyhidi, Jr., L., Tartar's conjecture and localization of the quasiconvex hull in $\mathbb{R}^{2 \times 2}$, Acta Math., to appear.

[8] Hurewicz, W., and Wallman, H., Dimension Theory, Princeton Math. Ser. 4, Princeton University Press, Princeton, N. J., 1941.

[9] Iwaniec, T., Verchota, G. C., and Vogel, A. L., The failure of rank-one connections, Arch. Rat. Mech. Anal. 163, 2 (2002), 125-169.

[10] Kinderlehrer, D., and Pedregal, P., Characterizations of Young measures generated by gradients, Arch. Rat. Mech. Anal. 115 (1991), 329-365.

[11] Kirchheim, B., Deformations with finitely many gradients and stability of quasiconvex hulls, C. R. Acad. Sci. Paris Sér. I Math. 332, 3 (2001), 289-294.

[12] Kirchheim, B., Rigidity and Geometry of microstructures, Habilitation thesis, University of Leipzig, 2003.

[13] Kirchheim, B., Müller, S., and Šverák, V., Studying nonlinear PDE by geometry in matrix space, in: Geometric analysis and nonlinear partial differential equations, S. Hildebrandt and H. Karcher, eds., Springer-Verlag (2003), 347-395. 
[14] Kuratowski, C., Topologie, Vol. II, Troisième édition, corrigée et complétée de deux appendices, Monogr. Mat. 21, Państwowe Wydawnictwo Naukowe, Warsaw 1961.

[15] Matos, J. P., Young measures and the absence of fine microstructures in a class of phase transitions, Europ. J. Appl. Math. 3, 1 (1992), 31-54.

[16] Müller, S., Variational models for microstructure and phase transitions, in: Calculus of variations and geometric evolution problems (Cetraro 1996), Lect. Notes Math. 1713, Springer (1999), 85-210.

[17] Pedregal, P., Parametrized measures and variational principles, Progr. Nonlin. Diff. Equ. Appl. 30, Birkhäuser Verlag, Basel 1997.

[18] Reshetnyak, Y. G., A condition for boundedness of the index for mappings with bounded distortion, Sibirsk. Mat. Ž. 9 (1968), 368-374.

[19] Reshetnyak, Y. G., The local structure of mappings with bounded distortion, Sibirsk. Mat. Ž. 10 (1969), 1311-1333.

[20] Reshetnyak, Y. G., The set of branch points of mappings with bounded distortion, Sibirsk. Mat. Ž. 11 (1970), 1333-1339.

[21] Rickman, S., Quasiregular mappings, Ergebn. Math. Grenzgeb. (3) 26, Springer-Verlag, Berlin 1993.

[22] Šverák, V., On regularity for the Monge-Ampère equations, preprint, Heriot-Watt University, 1991.

[23] Šverák, V., On Tartar's conjecture, Ann. Inst. H. Poincaré Anal. Non Lin. 10 (1993), 405-412.

[24] Székelyhidi, Jr., L., Rank-one convex hulls in $\mathbb{R}^{2 \times 2}$, Calc. Var. Part. Diff. Equ. 22, 3 (2005), $253-281$.

[25] Zhang, K., On connected subsets of $M^{2 \times 2}$ without rank-one connections, Proc. Roy. Soc. Edinburgh Sect. A 127, 1 (1997), 207-216.

[26] Zhang, K., Neighborhoods of parallel wells in two dimensions that separate gradient Young measures, SIAM J. Math. Anal. 34, 5 (2003), 1207-1225.

[27] Zhang, K., On separation of gradient Young measures, Calc. Var. Part. Diff. Equ. 17, 1 (2003), 85-103.

Mathematical Institute, Oxford University, 24-29 St. Giles', Oxford, OX1 3LB, UK

e-mail: kirchhei@maths.ox.ac.uk

Departement Mathematik, ETH Zürich, 8092 Zürich, Switzerland e-mail: szekelyh@math.ethz.ch

Eingegangen 23. März 2007, in revidierter Fassung 14. Januar 2008 